\title{
ANÁLISIS DE LA DIVERSIDAD GENÉTICA DE AGAVES MEZCALEROS DEL CENTRO DE MÉXICO
}

\author{
ANALYSIS OF GENETIC DIVERSITY OF MEZCALERO AGAVES FROM CENTRAL MÉXICO
}

\author{
José P. Lara-Ávila' y Ángel G. Alpuche-Solís
}

\begin{abstract}
'Facultad de Agronomía y Veterinaria, Universidad Autónoma de San Luis Potosí. Km. 14.5 Carr. San Luis Potosí-Matehuala. Apartado Postal 32, Ejido Palma de la Cruz. 78321, Soledad de Graciano Sánchez. S. L. P. México. ²División de Biología Molecular, Instituto Potosino de Investigación Científica y Tecnológica A. C. Camino a la Presa San José 2055, Apartado Postal 78216, Lomas 4ta. Sección, San Luis Potosí, S.L.P. México. Tel. +52 (444) 8342000.
\end{abstract}

*Autor de correspondencia (alpuche@ipicyt.edu.mx)

\section{RESUMEN}

En el Altiplano Potosino, un ecosistema árido y semiárido, Agave salmiana Otto Salm Dick ssp. crassispina (Trel Gentry) es una especie utilizada para la recolección de insectos comestibles y para producción de mezcal, bebida alcohólica tradicional con denominación de origen. El aprovechamiento para producción de mezcal de las poblaciones silvestres de Agave salmiana ssp. crassispina carece de un sistema de explotación basado en el conocimiento biológico de la especie. Esto tiene efectos demográficos, y a su vez podría causar el deterioro de sus recursos fitogenéticos y colocar a la especie en situación de riesgo. Este trabajo analizó diversidad genética y estructura poblacional de tres poblaciones silvestres de Agave salmiana ssp. crassispina en San Luis Potosí, México, mediante polimorfismos de longitud de los fragmentos amplificados, AFLP. La evidencia mostró un alto nivel de diversidad genética dentro de las poblaciones analizadas y un bajo nivel de diferenciación entre ellas, probablemente debido a una fragmentación del hábitat producida por actividades antropogénicas. Postulamos que la diversidad genética en las poblaciones analizadas, a pesar de la constante explotación, se origina por el sinergismo entre la polinización cruzada y la actividad de elementos genéticos transponibles. El alto grado de diversidad genética encontrado en las poblaciones silvestres analizadas en San Luis Potosí demuestra la importancia agroecológica de Agave salmiana ssp. crassispina en el Altiplano Mexicano, una región geográfica que abarca casi la mitad del territorio mexicano. Sin embargo, uso descontrolado y manejo inapropiado de las magueyeras silvestres podría arriesgar los recursos fitogenéticos de Agave salmiana ssp. crassispina.

Palabras clave: Agave salmiana ssp. crassispina, diversidad genética, ecosistemas áridos, marcadores moleculares.

\section{SUMMARY}

The Potosino Upland Plateau is an arid and semiarid ecosystem. Agave salmiana Otto Salm Dick ssp. crassispina (Trel Gentry), which grows in this environment, is a species used for gathering of edible insects and for production of mezcal. Mezcal is a Mexican alcoholic beverage with denomination of origin. Production of mezcal with wild populations of Agave salmiana ssp. crassispina lacks an exploitation system based on biological knowledge of the species. The lack of organized use has demographic effects, which leads to degradation of genetic resources and risks to this species. This study analyzed genetic diversity and population structure of three wild populations of Agave salmiana ssp. crassispina from San Luis Potosí, Mexico by AFLP's (amplified fragment length polymorphism). The evidence showed a high level of genetic diversity within populations and low level of genetic differentiation among populations, probably as a result of habitat fragmentation produced by anthropogenic activities. We postulate that the genetic diversity in the wild populations analyzed, despite their constant exploitation, is originated by the synergism between cross-pollination and the activity of transposable genetic elements. The high level of genetic diversity found in the wild populations in San Luis Potosí shows agro-ecological relevance of Agave salmiana ssp. crassispina in the Mexican upland plateau. However, uncontrolled use and inappropriate management of wild populations could risk the genetic resources of Agave salmiana ssp. crassispina.

Index words: Agave salmiana ssp. crassispina, genetic diversity, arid environment, molecular markers.

\section{INTRODUCCIÓN}

El género Agave es un habitante típico de los ecosistemas áridos y semiáridos de México, y posee un alto grado de endemismo. Aproximadamente $75 \%$ de las especies de Agave son endémicas, y algunas de ellas tienen relevancia antropogénica desde la época prehispánica (Aguirre-Rivera et al., 2001; Gentry, 1982; Parker et al., 2010; Zizumbo-Villarreal et al., 2013). La riqueza actual de especies en Agave se puede explicar en función del desarrollo de adaptaciones fisiológicas, morfológicas y ecológicas ocurridas hace 8 a 10 millones de años y que favorecieron que las nuevas especies de Agave colonizaran las recién formadas zonas áridas que corresponden al actual Altiplano Central Mexicano (Good-Avila et al., 2006; Gross et al., 2013; Shakeel et al., 2013).

Por otro lado, la relación Agave-Ser Humano permitió la aparición de otras especies y variantes con significancia socio-cultural (Aguirre-Rivera et al., 2001; Colunga-GarcíaMarin et al., 1996; Parker et al., 2010). Agave salmiana Otto Salm Dick ssp crassispina (Trel Gentry) es una de las especies adaptadas a hábitats áridos y semiáridos del Altiplano Mexicano. Las poblaciones silvestres de A. salmiana ssp. crassispina han sido utilizadas tradicionalmente para la elaboración de mezcal en Durango, San Luis Potosí y Zacatecas (Aguirre-Rivera et al., 2001; CONABIO, 2006; Martínez-Salvador et al., 2005; Martínez-Salvador et al., 2007), y para la recolección de insectos comestibles como el gusano blanco (Acentrocneme hesperiaris), gusano rojo 
(Comadia redtenbacheri) y escamoles (larvas de Liometopum apiculatum) (Esparza-Frausto et al., 2008).

En este contexto, la producción de mezcal requiere cosechar individuos en propagación vegetativa (Aguirre-Rivera et al., 2001; Zizumbo-Villarreal et al., 2013), lo cual limita la probabilidad de que se lleve a cabo la reproducción sexual, reduce la tasa de reproducción vegetativa (producción de hijuelos), y reduce el tamaño de poblaciones, lo cual se traduce en impactos negativos a nivel demográfico, ambiental y probablemente genético (Aguirre-Dugua y Eguiarte, 2013; Martínez-Salvador et al., 2005; Martínez-Salvador et al., 2007; Sánchez-Teyer et al., 2009). Actualmente algunas especies de Agave están consideradas en riesgo (Eguiarte et al., 2013).

La pérdida de la biodiversidad ocurre a través de la pérdida de especies y alelos. La disminución de la diversidad genética en las especies reduce los procesos de adaptación a cambios ambientales y afecta su supervivencia a largo plazo, lo cual incrementa el riesgo de extinción (Bourguiba et al., 2012; Huang et al., 2009). Por ello los estudios de diversidad genética son fundamentales para el desarrollo de estrategias de aprovechamiento sustentable de las especies con interés económico (Huang et al., 2009; Zizumbo-Villarreal et al., 2013).

El objetivo del presente trabajo fue evaluar la diversidad genética de A. salmiana ssp. crassispina, en tres poblaciones silvestres bajo explotación para la producción de mezcal en el Altiplano Potosino, mediante el análisis de la huella genética generada por marcadores moleculares AFLP.

\section{MATERIALES Y MÉTODOS}

\section{Material vegetal}

Se colectaron muestras de hoja de 19, 23 y 24 individuos de A. salmiana ssp. crassispina en tres poblaciones silvestres: Aquiles Serdán (AqS) (23 16' 52.74" N, 100 55' 12.08" O), El Cuervo (EC) (23 14' 59.81" N, $100^{\circ} 55^{\prime} 39.94^{\prime \prime}$ O), ambas localizadas en el municipio de Charcas, San Luis Potosí; e Ipiña (Ipi) (22 26' N, $101^{\circ}$ 19' 30" O) en el municipio de Ahualulco, San Luis Potosí. Las tres poblaciones muestreadas son explotadas para la producción de mezcal. La distancia entre los sitios Aquiles Serdán y El Cuervo es 1.5 km, y ambos sitios están ubicados a 101 km aproximadamente de Ipiña.

\section{Extracción de ADN}

El ADN genómico se extrajo con el siguiente protocolo. Se utilizaron $0.5 \mathrm{~g}$ de muestra de hoja y se congelaron por inmersión en nitrógeno líquido. La muestra se trituró en mortero hasta obtener un polvo fino, al cual se agregaron $500 \mu \mathrm{L}$ de buffer de extracción (D-sorbitol $0.14 \mathrm{M}$; Tris $\mathrm{HCl}$ $0.22 \mathrm{M}$; EDTA $0.022 \mathrm{M}$; NaCl $0.8 \mathrm{M}$; CTAB $0.8 \%$; n-lauril sarcosina $1 \%$; pH 8) en un tubo de $1.5 \mathrm{~mL}$ con agitación en vórtex. El tubo se incubó a $65^{\circ} \mathrm{C}$ por 30 min con agitación constante. Posteriormente, el tubo se centrifugó durante 10 min a 8510 Xg (MiniSpin®, Eppendorf, USA). El sobrenadante se separó y transfirió a un tubo nuevo.

A este tubo se adicionó un volumen igual de fenol/cloroformo/isoamílico (25/24/1), se mezcló en vórtex, y se centrifugó durante 10 min a 8510 Xg (MiniSpin, Eppendorf, USA). La fase acuosa se separó con pipeta y transfirió a un tubo nuevo de $1.5 \mathrm{~mL}$. La extracción con fenol/cloroformo/isoamílico (25/24/1) se repitió con el sobrenadante. La fase acuosa se transfirió a un tubo nuevo con $1 \mu \mathrm{L}$ de RNAasa $(1 \mu \mathrm{g} \mathrm{mL}-1)$ e incubada a $37^{\circ} \mathrm{C}$ por $45 \mathrm{~min}$. El ADN se precipitó con un volumen igual de isopropanol frío, y el tubo se incubó en hielo por 5 min. Posteriormente, el tubo se centrifugó durante 5 min a $8510 \mathrm{Xg}$, y el sobrenadante se eliminó por decantación. La pastilla de ADN se lavó gentilmente con etanol $70 \%$, seguido de un paso de centrifugación por 5 min a 8510 Xg. El etanol se eliminó con pipeta. La pastilla de ADN se dejó secar a temperatura ambiente y se resuspendió en $50 \mu \mathrm{L}$ de agua Milli $Q$ estéril.

\section{Marcadores moleculares AFLP}

El protocolo de marcadores moleculares AFLP se basó en Vos et al. (1995). Aproximadamente $250 \mathrm{ng}$ de ADN se digirieron simultáneamente con las enzimas de restricción EcoRI y Msel, seguido de una ligación de adaptadores específicos con T4 ADN ligasa (Invitrogen). Los fragmentos ligados fueron utilizados como templetes para una preamplificación con los oligonucleótidos preselectivos EcoRI + A (5'-AGA CTG CGT ACC AAT TCA-3') y Msel + C (5'-GAC GAT TCC TGA GTA AC-3'). Para la amplificación selectiva se utilizó la combinación de oligonucleótidos Msel + CTG (5'-GAC GAT GAG TCC TGA GTA ACT G-3') y ECORI + ACA (5'-AGA CTG CGT ACC AAT TCA CA-3'). El oligonucleótido ECoRI + ACA se etiquetó con fluorescencia (IRDye 800). Los marcadores moleculares AFLP se separaron en un gel de poliacrilamida en condiciones desnaturalizantes (PAGE 6 \%) en un secuenciador automático LiCor 4200L (LI-CORß Biosciences; Lincoln, NE, USA).

\section{Análisis de datos}

Los marcadores moleculares AFLP se analizaron asumiendo que: i) las bandas de ADN correspondieron a un locus genético y que los alelos marcadores de diferentes loci no co-emigran a la misma posición en el gel de electroforesis; ii) que cada locus puede ser tratado como un sistema de dos alelos, y se trata de un alelo amplificable 
por PCR; y iii) que las poblaciones están en el equilibrio de Hardy-Weinberg (Lynch y Milligan, 1994). La diversidad genética se midió mediante el cálculo de medidas basadas en distancia y en frecuencias alélicas, dentro de cada población y entre ellas, a partir de la matriz binaria de presencia / ausencia de bandas-alelos (1 / 0) (Bonin et al., 2007). La matriz binaria se obtuvo a partir del patrón de bandeo del gel de AFLP mediante el "software" Cross Checker V2.9 (Buntjer y Otsen, 1999).

Los marcadores moleculares dominantes pueden tener una sobreestimación de aproximadamente $5 \%$ en los parámetros de diversidad, principalmente en aquellos donde el número de muestras es relativamente pequeño (Lynch y Milligan, 1994), por lo cual se eliminaron aquellos loci con frecuencia de banda mayor que 1 - $(3 / N)$, donde $N=$ número de individuos muestreados. La frecuencia de cada banda-alelo se calculó con el "software" GenALEX versión 6.5 (Peakell y Smouse, 2012) para cada población. El cálculo de los parámetros de diversidad se realizó con el conjunto de los datos completos y los corregidos. Los datos obtenidos con la corrección de Lynch y Milligan (1994) se reportan en el presente trabajo.

La diversidad genética a nivel de poblaciones individuales se midió mediante el cálculo del número de alelos $\left(n_{a}\right)$, el número efectivo de alelos $\left(n_{e}\right)$ (Kimura y Crow, 1964), la diversidad genética no sesgada de Nei (Nei, 1978; Nei, 1987) (uHe) y el índice de Shannon (I) (Lewontin, 1972) mediante el "software" Popgene 1.32 (Yeh et al., 1999) y GenALEX versión 6.5 (Peakell y Smouse, 2012). A nivel de grupo de poblaciones, el parámetro diversidad genética total en todas las poblaciones $(\mathrm{Ht})$, media de diversidad genética dentro de las poblaciones $(H s)$, diferenciación de poblaciones (Gst) y el estimado de flujo génico (Nm), se calcularon con el "software" Popgene 1.32 al considerar un grupo con tres poblaciones (Aquiles Serdán, El Cuervo, Ipiña) y un grupo con dos poblaciones (Aquiles Serdán, El Cuervo).

Un dendrograma basado en el coeficiente de Jaccard con el método UPGMA con 1000 permutaciones de "bootstrap" (técnica de remuestreo no paramétrico), se calculó con el "software" FreeTree (Bonin et al., 2007; Hampl et al., 2001). La partición de la variación en dos niveles, entre poblaciones y dentro de poblaciones, mediante el análisis molecular de varianza (AMOVA), se calculó con base en una matriz de distancia euclidiana con el "software" Arlequin v3.5 (Excoffier et al., 2010). La significancia de los componentes de varianza se probó con 1000 permutaciones.

\section{RESULTADOS Y DISCUSIÓN}

La combinación de oligonucleótidos ECoRI+ACA y Msel+CTG, generó un patrón de 91 loci en 66 individuos muestreados de A. salmiana ssp. crassispina. Al aplicar la corrección de Lynch y Milligan (1994), el porcentaje de loci polimórficos dentro de cada población fue 96.7 \%, $98.9 \%$ y $78.02 \%$ (Cuadro 1).

\section{Diversidad, diferenciación y estructura genética en poblaciones silvestres de $A$. salmiana ssp. crassispina en el Altiplano Potosino}

Los resultados de diversidad genética no sesgada $(\mathrm{uHe})$ mostraron valores similares entre las poblaciones AqS y EC $(0.448 \pm 0.012 ; 0.42 \pm 0.011)$, mientras que la población Ipi mostró niveles más bajos (0.34 \pm 0.02$)$. Al formar un grupo con las poblaciones AqS + EC, en función de su cercanía geográfica y considerarla como una única población, y otro grupo considerando las tres poblaciones AqS + $\mathrm{EC}+$ Ipi, los valores de uHe mostraron diferencias (0.434 \pm $0.008 ; 0.403 \pm 0.0092$ ) entre los grupos formados.

Sin embargo, el índice de información de Shannon (I) mostró valores similares a nivel de poblaciones individuales y a nivel de grupos. La diferencia entre estos parámetros radica en que uHe es una métrica basada en el cálculo de frecuencias alélicas aplicable a una muestra de tamaño reducido, mientras que I es una métrica basada en la comparación apareada del perfil de bandeo entre individuos (Bonin et al., 2007). La información que brinda / es por lo

Cuadro 1. Parámetros de diversidad genética al interior de las poblaciones de $A$. salmiana ssp. crassispina.

\begin{tabular}{lcccccc}
\hline Población & Tamaño & $n_{a}$ & $n_{e}$ & $u H e$ & $l$ & $\%$ LP \\
\hline AqS & 19 & 2 & $1.8469 \pm 0.1973$ & $0.448 \pm 0.012$ & $0.6401 \pm 0.0885$ & 96.7 \\
EC & 23 & 2 & $1.7451 \pm 0.2289$ & $0.420 \pm 0.011$ & $0.6023 \pm 0.1017$ & 98.9 \\
Ipi & 24 & 2 & $1.7725 \pm 0.2049$ & $0.340 \pm 0.020$ & $0.6158 \pm 0.0848$ & 78.0 \\
AqS+EC & 41 & 2 & $1.8708 \pm 0.1367$ & $0.434 \pm 0.008$ & $0.6543 \pm 0.0451$ & 100 \\
AqS+EC+Ipi & 60 & 2 & $1.8740 \pm 0.1368$ & $0.403 \pm 0.009$ & $0.6552 \pm 0.0454$ & 100 \\
\hline
\end{tabular}

$n_{2}=$ número observado de alelos; $n_{e}=$ número efectivo de alelos (Kimura y Crow, 1964); uHe = diversidad genética no sesgada de Nei (Nei, 1978; Nei, 1983); I = índice de información de Shannon (Lewontin, 1972); \% LP = porcentaje de loci polimórficos. 
tanto de carácter fenotípico al considerar que los patrones de bandeo obtenidos por AFLP corresponden a un fenotipo molecular, mientras que uHe brinda información acerca del genotipo de la especie. Entonces, los resultados sugieren que las tres poblaciones de A. salmiana ssp. crassispina ubicadas en el Altiplano Potosino poseen diferencias en los niveles de diversidad genética, incluso entre las dos poblaciones cercanas geográficamente (Cuadro 1).

A nivel de grupo de poblaciones, los valores de Ht mostraron diferencias significativas al formar los grupos AqS $+E C+I P$ y AqS + EC (0.2598 $\pm 0.1129 ; 0.3827 \pm 0.1455)$. Los valores de Hs fueron diferentes entre los dos grupos $(0.3934 \pm 0.0043 ; 0.4235 \pm 0.0044)$. Por otro lado, los valores de diferenciación de poblaciones (Gst) fueron negativos al conjuntar las tres poblaciones (-0.5142) y al conjuntar dos poblaciones (-0.1067), y éstas deben considerarse como cero (Cuadro 2). Al respecto, el valor del estimado de flujo génico $(\mathrm{Nm})$, calculado a partir de Gst, tiende al infinito, lo cual sugiere una tasa de migración entre las poblaciones analizadas. Los resultados sugieren que entre las tres poblaciones de A. salmiana ssp. crassispina ubicadas en el Altiplano Potosino no hay diferenciación génica, aun cuando están separadas geográficamente.

\section{Estructura genética de las poblaciones silvestres analizadas de $A$. salmiana ssp. crassispina en el Altiplano Potosino}

Para visualizar las relaciones genéticas entre las poblaciones se calculó un dendrograma con el coeficiente de Jaccard mediante el método UPGMA y con 1000 permutaciones de "bootstrap" para estimar su robustez estadística (Bonin et al., 2007). El dendrograma mostró la tendencia de agrupar a los individuos de acuerdo con su origen geográfico: la población de Ipiña tuvo dos subgrupos, mientras que las poblaciones Aquiles Serdán y El Cuervo tuvieron cuatro subgrupos con diferente número de individuos en cada uno. Los subgrupos se distribuyeron en todo el dendrograma.

Algunos nodos del dendrograma muestran valores significativamente altos de "bootstrap", mientras que otros no- dos mostraron valores bajos. Esto puede explicarse por el alto grado de polimorfismo de los marcadores que apoyan la aparición de esos nodos con bajo valor de "bootstrap" en las tres poblaciones de A. salmiana ssp. crassispina; sin embargo, la topología general del árbol es más informativa que una rama en particular (Figura 1) (Mohammadi y Prassana, 2003).

Para cuantificar la diversidad genética revelada a nivel entre poblaciones y entre individuos al interior de las poblaciones de A. salmiana ssp. crassispina, se calculó el análisis molecular de varianza (AMOVA). Este análisis reveló que 88.73 \% de la variación genética total se ubica entre individuos al interior de las poblaciones, mientras que $11.27 \%$ se ubica entre poblaciones. El parámetro de diferenciación de poblaciones $\left(F_{\mathrm{ST}}\right)$ estimado de AMOVA tuvo un valor 0.11266 (Cuadro 3). Los resultados confirman que existe un bajo nivel de diferenciación genética poblacional entre las tres poblaciones de A. salmiana ssp. crassispina.

En resumen, el análisis de marcadores moleculares AFLP en tres poblaciones silvestres de A. salmiana ssp. crassispina, sugiere la existencia de un alto grado de variabilidad genética entre individuos dentro de las poblaciones, y un bajo nivel de diferenciación genética entre las tres poblaciones analizadas. Al respecto, las poblaciones Aquiles Serdán y El Cuervo mostraron niveles similares de diversidad genética $(u H e)$, probablemente debido a su cercanía geográfica.

\section{Consideraciones agroecológicas de la diversidad genética en las tres poblaciones silvestres de A. salmiana ssp. crassispina}

Las tres poblaciones analizadas de A. salmiana ssp. crassispina son explotadas para la elaboración de mezcal en el Altiplano Potosino. Dicha práctica requiere la extracción de individuos en estado de propagación vegetativa (Aguirre-Rivera et al., 2001; Martínez-Salvador et al., 2005). La existencia de altos niveles de diversidad genética en varias especies de Agave con propagación vegetativa ha sido reportada previamente (Aguirre-Dugua y Eguiarte, 2013; Díaz-Martínez et al., 2012; Eguiarte et al., 2013; Infante et al., 2006).

Cuadro 2. Parámetros de diversidad y diferenciación genética entre poblaciones de las poblaciones silvestres de $A$. salmiana subsp. crassispina.

\begin{tabular}{llllll}
\hline & Tamaño & $H t$ & $H s$ & Gst & $N m$ \\
\hline AqS+EC+Ipi & 60 & $0.2598 \pm 0.1129$ & $0.3934 \pm 0.0043$ & -0.5142 & $\infty$ \\
AqS+EC & 42 & $0.3827 \pm 0.1455$ & $0.4235 \pm 0.0044$ & -0.1067 & $\infty$ \\
\hline
\end{tabular}

$H t=$ diversidad genética total de todas las poblaciones; $H s=$ media de la diversidad genética al interior de las poblaciones; Gst = diferenciación genética de poblaciones, a partir de Gst $=(\mathrm{Ht}-\mathrm{Hs}) / \mathrm{Ht} ; \mathrm{Nm}=$ estimado del flujo génico, a partir de Gst; Nm = 0.5(1 - Gst) / Gst (Balloux y LugonMoulin, 2002; McDermott y McDonald, 1993). 


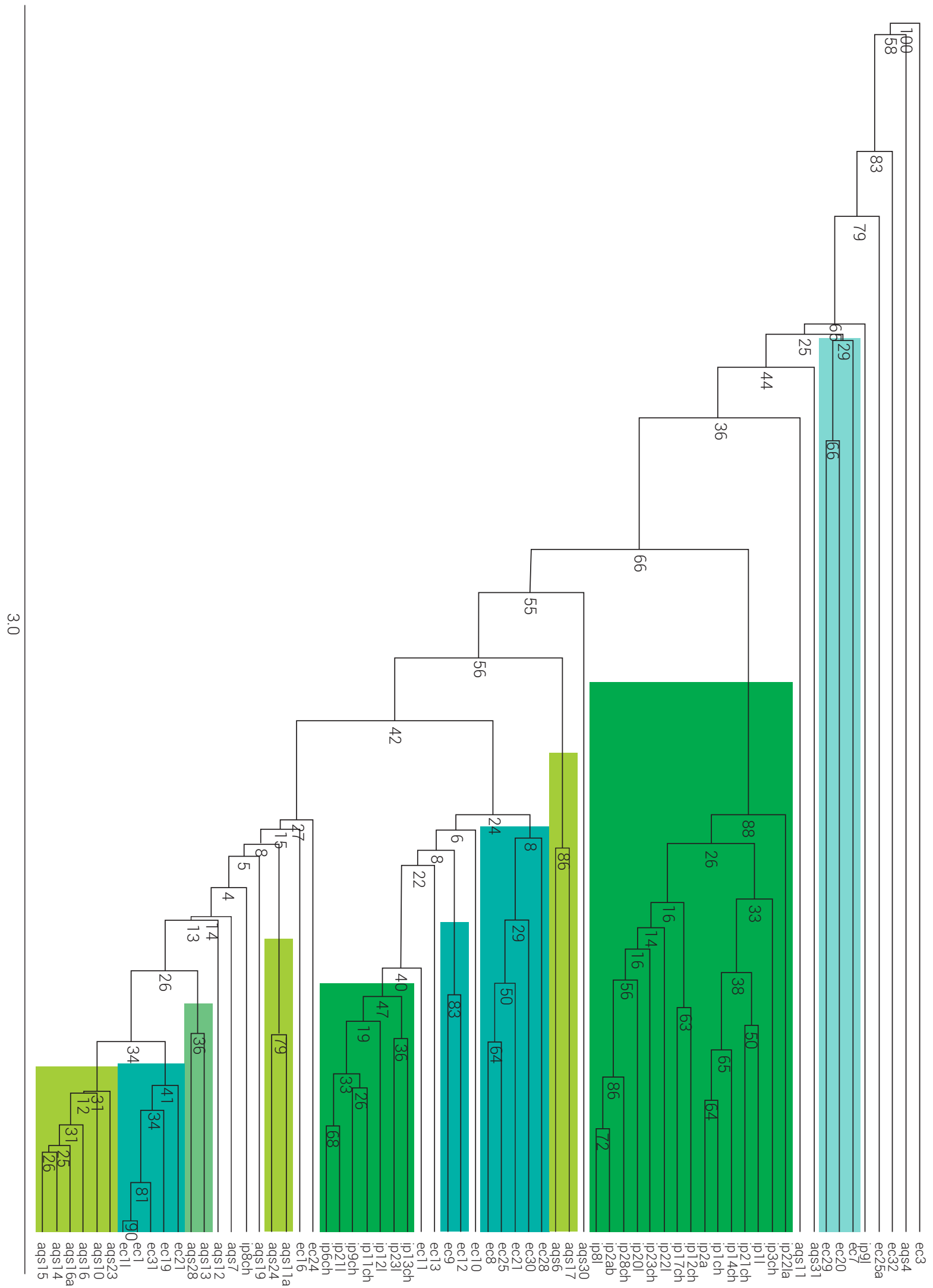

Figura 1. Análisis de agrupamiento jerárquico de $A$. salmiana ssp. crassispina basado en el coeficiente de Jaccard con el método UPGMA. Los valores de "bootstrap" se ubican en cada nodo del dendrograma. Los individuos con clave "aqs" pertenecen a la población Aquiles Serdán, "ec" pertenecen a la población El Cuervo; "ip" pertenecen a la población Ipiña. 
Cuadro 3. Análisis molecular de varianza (AMOVA) en poblaciones silvestres de $A$. salmiana subsp. crassispina.

\begin{tabular}{lcccc}
\hline Fuente de variación & Grados de libertad & Suma de cuadrados & Componentes de varianza & Porcentaje de variación \\
\hline Entre poblaciones & 2 & 104.26 & 1.75108 & 11.27 \\
Dentro de poblaciones & 63 & 868.891 & 13.79193 & 88.73 \\
Total & 65 & 973.152 & 15.54301 & 100 \\
$F_{\text {ST }} 0.11266$ & & & \\
\hline
\end{tabular}

En este contexto, se ha documentado que la propagación vegetativa en A. tequilana y A. duranguensis está asociada a variabilidad epigenética heredable, que incluye la actividad de retrotransposones pertenecientes a las familias Ty1-copia; Gypsy y CACTA, así como a diferencias en los patrones de metilación entre plantas madre y sus respectivos hijuelos (Díaz-Martínez et al., 2012; Gross et al., 2013; Khaliq et al., 2012). Asimismo, se ha descrito la relevancia de la variabilidad epigenética en los procesos evolutivos de las especies vegetales, al presentar un comportamiento diferencial en tejidos o en presencia de estímulos abióticos y bióticos (Díaz-Martínez et al., 2012; Richards, 2006; Schnable et al., 2009). Por ello, la variabilidad epigenética contribuye significativamente en la generación de variabilidad natural de las especies vegetales (Fujimoto et al., 2012; Khaliq et al., 2012; Torres-Morán et al., 2012; Zhang y Hsieh, 2013).

Este trabajo muestra que el alto grado de diversidad genética encontrada en las tres poblaciones silvestres analizadas de A. salmiana ssp. crassispina, podría ser el resultado del sinergismo entre un componente de variabilidad genética heredable (polinización cruzada) y un componente epigenético heredable, el cual incluiría la actividad de elementos genéticos transponibles y diferencias en patrones de metilación entre plantas madre y sus hijuelos.

Con base en la información de diversidad genética disponible, se han identificado especies como $A$. angustifolia y $A$. victoriae-reginae que necesitan esfuerzos prioritarios para su conservación. Estas especies poseen altos grados de diversidad genética al interior de cada especie y de diferenciación entre poblaciones, por lo cual, para salvaguardar el mayor número de alelos de cada especie se requieren altos números de individuos y poblaciones (Eguiarte et al., 2013). Según los resultados de diversidad genética de las tres poblaciones analizadas en este estudio, los esfuerzos de conservación de A. salmiana ssp. crassispina deberían estar dirigidos a resguardar tantos individuos como sea posible sin considerar muchas poblaciones (Eguiarte et al., 2013).

Tomando en cuenta los valores de flujo génico entre las tres poblaciones analizadas de A. salmiana ssp. cras- sispina, los datos sugieren que las tres poblaciones se comportan como una sola población. En este escenario, los resultados de flujo génico deberían ser interpretados como evidencia de migraciones históricas entre las tres poblaciones actuales, las cuales derivarían de una sola población, que pudo haber sido fragmentada por actividades antropogénicas relativamente recientes (Habel et al., 2015; Li et al., 2015; Luan et al., 2006; Zhao et al., 2012).

A pesar de la pérdida de hábitats y del efecto demográfico negativo como consecuencia de la extracción de individuos en propagación vegetativa (Martínez-Salvador et al., 2005; 2007), el alto nivel de variabilidad genética en las tres poblaciones analizadas de A. salmiana ssp. crassispina, una especie ampliamente distribuida en el Altiplano Mexicano, asegura la compatibilidad entre la conservación de la especie y su aprovechamiento sustentable. Asimismo, el hecho resalta la necesidad de desarrollar enfoques para la protección de los recursos fitogenéticos endémicos con alto valor social y agroecológico (Eguiarte et al., 2013; Martínez-Salvador et al., 2007; Mora-Lopez et al., 2011).

\section{CONCLUSIONES}

A. salmiana ssp. crassispina es una especie ampliamente distribuida en el Altiplano Mexicano, que ha sufrido pérdida de hábitat y el efecto demográfico negativo como consecuencia de la extracción de individuos en propagación vegetativa. Este estudio demuestra el alto nivel de variabilidad genética en las tres poblaciones analizadas de A. salmiana ssp. crassispina. La variabilidad identificada asegura la compatibilidad entre la conservación de la especie y su aprovechamiento sustentable. Estos resultados sustentan la necesidad de desarrollar enfoques para la protección de los recursos fitogenéticos endémicos con alto valor social y agroecológico.

\section{AGRADECIMIENTOS}

El presente estudio se realizó en el Laboratorio de Biología Molecular de Plantas perteneciente al Instituto Potosino de Investigación Científica y Tecnológica A. C. en colaboración con la Facultad de Agronomía y Veterinaria de la Universidad Autónoma de San Luis Potosí. Los autores agradecen al Consejo Nacional de Ciencia y Tecnología 
(CONACYT apoyo No. 202399), a Verónica Zárate-Chávez y Rosalba Castillo-Collazo por su apoyo técnico y a los revisores por las valiosas críticas para la mejora de este manuscrito.

\section{BIBLIOGRAFÍA}

Aguirre-Dugua X. and L. E. Eguiarte (2013) Genetic diversity, conservation and sustainable use of wild Agave cupreata and Agave potatorum extracted for mezcal production in Mexico. Journal of Arid Environments 90:36-44

Aguirre-Rivera J. R., H. Charcas-Salazar y J. L. Flores-Flores (2001) El Maguey Mezcalero Potosino. Consejo Potosino de Ciencia y Tecnología, Universidad Autónoma de San Luis Potosí, San Luis Potosí. $78 \mathrm{p}$.

Balloux F. and N. Lugon-Moulin (2002) The estimation of population differentiation with microsatellite markers. Molecular Ecology 11:155-165

Bonin A., D. Ehrich and S. Manel (2007) Statistical analysis of amplified fragment length polymorphism data: a toolbox for molecular ecologists and evolutionists. Molecular Ecology 16:3737-3758.

Bourguiba H., J. M. Audergon, L. Krichen, N. Trifi-Farah, A. Mamouni, S. Trabelsi, C. D'Onofrio, B. M. Asma, S. Santoni and B. Khadari (2012) LosS of genetic diversity as a signature of apricot domestication and diffusion into the Mediterranean Basin. BMC Plant Biology 12:49.

Buntjer J. B. and M. Otsen (1999) Cross Checker provides computer-assisted marker interpretation. Journal of Agricultural Genomics 4:1-6.

Colunga-GarcíaMarin P., E. Estrada-Loera and F. May-Pat (1996) Patterns of morphological variation, diversity, and domestication of wild and cultivates populations of Agave in Yucatán México. American Journal of Botany 83:1069-1082.

CONABIO, Comisión Nacional para el Conocimiento y Uso de la Biodiversidad (2006) Mezcales y Diversidad. 2a. ed. Comisión Nacional para el Conocimiento y Uso de la Biodiversidad. México, D.F. (Mapa).

Díaz-Martínez M., A. Nava-Cedillo, J.A. Guzmán-López, R. Escobar-Guzmán and J. Simpson (2012) Polymorphism and methylation patterns in Agave tequilana Weber var. Azul plants propagated asexually by three different methods. Plant Science 185-186:321-330.

Eguiarte L. E., E. Aguirre-Planter, X. Aguirre, R. Colín, A. González, M. Rocha, E. Scheinvar, L. Trejo and V. Souza (2013) From isozymes to genomics: population genetics and conservation of Agave in México. The Botanical Review 79:483-506.

Esparza-Frausto G., F. J. Macías-Rodríguez, M. Martínez-Salvador, M. A. Jiménez- Guevara y S. J. Méndez-Gallegos (2008) Insectos comestibles asociados a las magueyeras en el ejido Tolosa, Pinos, Zacatecas, México. Agrociencia 42:243-252.

Excoffier L. and H. E. L. Lischer (2010) Arlequin suite ver 3.5: a new series of programs to perform population genetics analyses under Linux and Windows. Molecular Ecology Resources 10:564-567.

Fujimoto R., T. Sasaki, R. Ishikawa, K. Osabe, T. Kawanabe and E. S. Dennis (2012) Molecular Mechanisms of Epigenetic Variation in Plants. International Journal of Molecular Sciences 13:9900-9922.

Gentry H. S. (1982) Agaves of Continental North America. The University of Arizona Press, Tucson.

Good-Avila S.V., V. Souza, B. S. Gaut and L. E. Eguiarte (2006) Timing and rate of speciation in Agave (Agavaceae). Proceedings of the National Academy of Sciences of the United States of America 103:9124-9129.

Gross S. M., J. A. Martin, J. Simpson, M. J. Abraham-Juarez, Z. Wang and A. Visel (2013) De novo transcriptome assembly of drought tolerant CAM plants, Agave deserti and Agave tequilana. BMC Genomics 14:563

Habel J. C., S. V. Brückmann, J. Krauss, J. Schwarzer, A. Weig, M. Husemann and I. Steffan-Dewenter (2015) Fragmentation genetics of the grassland butterfly Polyommatus coridon: Stable genetic diversity or extinction debt? Conservation Genetics 16:549-558

Hampl V., A. Pavlícek and J. Flegr (2001) Construction and bootstrap analysis of DNA fingerprinting-based phylogenetic trees with the freeware program FreeTree: application to trichomonad parasites. International Journal of Systematic and Evolutionary Microbiology 51:731-735

Huang Y., C. Q. Zhang and D. Z. Li (2009) Low genetic diversity and high genetic differentiation in the critically endangered Omphalogramma souliei (Primulaceae): implications for its conservation. Journal of Systematics and Evolution 47:103-109.

Infante D., S. Molina, J. R. Demey and E. Gámez (2006) Asexual genetic variability in Agavaceae determined with inverse sequence-tagged repeats and amplification fragment length polymorphism analysis. Plant Molecular Biology Reporter 24:205-217.

Khaliq I., M. A. Khan and S. Pearce (2012) Ty1-copia retrotransposons are heterogeneous, extremely high copy number and are major players in the genome organization and evolution of Agave tequilana. Genetic Resourses and Crop Evolution 59:575-587

Kimura M. and J. F. Crow (1964) The number of alleles that can be maintained in a finite population. Genetics 49:725-738.

Lewontin R. C. (1972) The apportionment of human diversity. Evolutionary Biology 6: 381-398.

Li Y., M. L. Lancaster, S. J. Cooper, A. C. Taylor and S. M. Carthew (2015) Population structure and gene flow in the endangered southern brown bandicoot (Isoodon obesulus obesulus) across a fragmented landscape. Conservation Genetics 16:331-345

Luan S., T. Y. Chiang and X. Gong (2006) High genetic diversity vs. low genetic differentiation in Nouelia insignis (Asteraceae), a narrowly distributed and endemic species in China, revealed by ISSR fingerprinting. Annals of Botany 98:583-589.

Lynch M. and B. G. Milligan (1994) Analysis of population genetic structure with RAPD markers. Molecular Ecology 3:91-99

Martínez-Salvador M., H. Rubio-Arias and A. Ortega-Rubio (2005) Population structure of maguey (Agave salmiana ssp. crassispina) in southeast Zacatecas, Mexico. Arid Land Research and Management 19:101-109.

Martínez-Salvador M., L. Beltrán-Morales, R. Valdéz-Cepeda, H. Arias-Rubio, E. Troyo-Diegez, B. Murillo-Amador, J. Galindo-Jiménez and A. OrtegaRubio (2007) Assessment of sustainability performance on the utilization of Agave (Agave salmiana ssp. crassispina) in Zacatecas, México. International Journal of Sustainable Development \& World Ecology 14:362-371

McDermott J. M. and B. A. McDonald (1993) Gene flow in plant pathosystems. Annual Review of Phytopathology 31:353-373

Mohammadi S. A. and B. M. Prassana (2003) Analysis of Genetic Diversity in Crop Plants - Salient Statistical Tools and Considerations. Crop Science 43:1235-1248.

Mora-López J. L., J.A. Reyes-Agüero, J. L. Flores-Flores, C. B. Peña-Valdivia y J. R. Aguirre-Rivera (2011) Variación morfológica y humanización de la sección Salmianae del género Agave. Agrociencia 45:465467.

Nei M. (1978) Estimation of average heterozygosity and genetic distance from a small number of individuals. Genetics 89:583-590.

Nei M. (1987) Molecular Evolutionary Genetics. Columbia University Press, New York. 512 p.

Parker K. C., D. W. Trapnell, J. L. Hamrick, W. C. Hodgson and A. J. Parker (2010) Inferring ancient Agave cultivation practices from contemporary genetic patterns. Molecular Ecology 19:1622-37.

Richards E. J. (2006) Inherited epigenetic variation - Revisiting soft inheritance. Nature Reviews Genetics 7:395-401.

Sánchez-Teyer F., S. Moreno-Salazar, M. Esqueda, A. Barraza and M. L. Robert (2009) Genetic variability of wild Agave angustifolia populations based on AFLP. A basic study for conservation. Journal of Arid Environments 73:611-616.

Schnable P. S., D. Ware, R. S. Fulton, J. C. Stein, F. Wei, S. Pasternak, C. Liang, J. Zhang, L. Fulton and T. A. Graves (2009) The b73 maize genome: Complexity, diversity, and dynamics. Science 326: 1112-1115.

Shakeel S.N., S. Aman, N.U. Haq, S.A. Heckathorn and D. Luthe (2013) Proteomic and tanscriptomic analyses of Agave americana in response to heat stress. Plant Molecular Biology Reporter 31:840851.

Torres-Morán M. I., N. Almaraz-Abarca and M. Escoto-Delgadillo (2012) ISTR, a Retrotransposons-based marker to assess plant genome variability with special emphasis in the genera Zea and Agave. American Journal of Plant Sciences 3:1820-1826

Vos P., R. Hogers, M. Bleeker, M. Reijans, T. van de Lee, M. Hornes, A. Frijters, J. Pot, J. Peleman and M. Kuiper (1995) AFLP. a new technique for 
DNA fingerprinting. Nucleic Acids Research 23:4407-4414

Yeh F. C., R. C. Yang and T. Boyle (1999) POPGENE. Microsoft Windowsbased freeware for population genetic analysis, release 1.31. University of Alberta, Edmonton, Alberta, Canada.

Zhang C. and T. F. Hsieh (2013) Heritable epigenetic variation and its potential applications for crop improvement. Plant Breeding and Biotechnology 1:307-319.

Zhao X., Y. Ma, W. Sun, X. Wen and R. Milne (2012) High genetic diversity and low differentiation of Michelia coriacea (Magnoliaceae), a critically endangered endemic in Southeast Yunnan, China. International Journal of Molecular Sciences 13:4396-411.

Zizumbo-Villarreal D., O. Vargas-Ponce, J. J. Rosales-Adame and P. ColungaGarcíaMarin (2013) Sustainability of the traditional management of Agave genetic resources in the elaboration of mezcal and tequila spirits in western Mexico. Genetic Resources and Crop Evolution 60:33-47. 